\title{
Análisis y Validación de la Consistencia en un Simulador de Conducción
}

\author{
Francisco Javier Camacho-Torregrosa \\ Grupo de Investigación en Ingeniería de Carreteras (GIIC), Universitat Politècnica de \\ València \\ Javier Marín-Morales \\ LabHuman, Universitat Politècnica de València \\ David Llopis-Castelló \\ GIIC, Universitat Politècnica de València
}

Ana María Pérez-Zuriaga

GIIC, Universitat Politècnica de València

Alfredo García

GIIC, Universitat Politècnica de València

\section{RESUMEN}

Muy diversas investigaciones previas han mostrado cómo la percepción del riesgo afecta el comportamiento de los conductores. Muchas de ellas van dirigidas a observar la diferencia de percepción entre conductores experimentados y noveles, o entre el riesgo objetivo y el percibido. Mientras que el riesgo objetivo puede ser obtenido a partir de las estadísticas de siniestralidad, el riesgo percibido es más complicado de evaluar. Existen diversos métodos, yendo desde la encuesta directa hasta técnicas de rastreo de la visión.

En este estudio se presenta una nueva metodología para recoger datos de Percepción Subjetiva de Riesgo (PSR) de forma quasi-naturalística. Con esta metodología, los conductores pueden indicar de forma sencilla su percepción de confort tras recorrer cada una de las curvas que compone un trazado. Posteriormente, la correlación de estos datos con la geometría de las curvas y otras variables permite derivar sencillas reglas para obtener un diseño consistente de la carretera. Igualmente, si esta metodología es válida para ser llevada a cabo en un entorno simulado, permitiría evaluar carreteras en fase de proyecto, convirtiéndose en una valiosa herramienta para los ingenieros.

Un total de 28 conductores participaron en la toma de datos, recorriendo el mismo tramo de carretera en la realidad y en entorno simulado, de $32 \mathrm{~km}$. En ambos casos indicaron su percepción de PSR tras cada una de las curvas aisladas. En este estudio se muestran los resultados alcanzados, tanto en el entorno real como el simulado. Igualmente, se establecen conexiones y correlaciones entre ambos métodos, mostrando bajo qué condiciones el entorno simulado arroja valores válidos. 


\section{INTRODUCCIÓN}

El factor humano es uno de los tres factores fundamentales en el análisis de los motivos que subyacen en la generación de accidentes, junto con la infraestructura y el vehículo. De ahí que sea necesario considerar dicho factor y su interacción con la infraestructura en aras de lograr unas carreteras más seguras.

La simulación de conducción es una herramienta muy interesante para incorporar este factor. Mediante los simuladores de conducción, pueden generarse escenarios virtuales, que serán recorridos por conductores y de los que se derivarán conclusiones que estarán relacionadas con la realidad.

La utilización de simuladores en fase de diseño permite evaluar diferentes alternativas, eligiendo la que presente una mejor acogida por parte de los conductores. En fase de explotación, permiten una toma de datos menos costosa y más precisa en cuanto a los parámetros recogidos. La contrapartida en ambos casos estriba en la precisión de dichos simuladores, tanto en cuanto al entorno simulado como a la fiabilidad del recorrido que el simulador hace por el mismo. Al proceso de determinar la fiabilidad de los simuladores con la realidad se le conoce como "validación" (Bella, 2009 y Kaptein et al., 1996). Esta validación consiste en determinar el grado de similitud entre la realidad y el simulador. Habitualmente se divide en:

- Validación objetiva. Comparación directa de los datos simulados con los recogidos en campo.

- Validación subjetiva. Evaluar la percepción de los conductores.

Por otra parte, muchos investigadores han reflejado que los conductores modifican su comportamiento en función del riesgo que perciben (Fuller, 2005, Näätänen y Summala, 1976). Además, Armsby et al. (1989) indican que el riesgo percibido es el más importante de los tres factores asociados a los accidentes de tráfico.

Kanellaidis y Dimitropoulos (1994) compararon la percepción subjetiva de riesgo en cinco curvas de Atenas, hallando una importante correspondencia con la siniestralidad. Sin embargo, en la curva con mayor siniestralidad no encontraron esa elevada percepción de riesgo.

Posteriormente, Kanellaidis et al. (2000) pusieron de relevancia la diferencia de percepción de riesgo en función de la edad, experiencia al volante, conocimiento de la vía, etc.

Otros estudios más recientes y con mejores medios, como el llevado a cabo por Charlton et al. (2014) utilizan nuevas técnicas, como el Eye-Tracking, para analizar los patrones de visualización en función del nivel de riesgo objetivo del elemento.

La percepción subjetiva de riesgo combinada con simulación de conducción podría utilizarse 
como una medida sustitutiva de la seguridad vial, con el objetivo de detectar, ya en fase de diseño, los posibles problemas de un trazado y así subsanarlo.

\section{OBJETIVOS E HIPÓTESIS}

El objetivo de la presente investigación es determinar si es posible utilizar un simulador de conducción para evaluar la Percepción Subjetiva de Riesgo (PSR) como medida sustitutiva de la seguridad vial. En dicho caso, se pretende obtener relaciones entre ciertas características geométricas y dicha variable.

Para ello se partirá de la hipótesis de que los conductores pueden indicar su percepción subjetiva de riesgo en cada curva de forma casi naturalística, no afectando a su conducción. Igualmente, se asumirá que dicha percepción tiene relación con el diseño geométrico y la consistencia de la misma, y que una mayor PSR está ligada a una mayor inconsistencia.

\section{METODOLOGÍA Y TOMA DE DATOS}

El proceso seguido en la investigación consiste en contrastar la Percepción Subjetiva de Riesgo de una serie de curvas con sus variables geométricas, tanto en la realidad como en simulador. Para ello se ha seleccionado un tramo de carretera de unos $30 \mathrm{~km}$ de longitud y una muestra de más de 20 conductores. El tramos será recorrido en ambos sentidos de circulación, tanto en la realidad como en un entorno simulado. En ambos casos, indicarán tras cada curva su PSR.

El tramo de carretera sobre el que se va a basar el estudio pertenece a la carretera CV-35, entre los municipios de Losa del Obispo y Titaguas (PK 53+500 a 83+700), en la provincia de Valencia.

Se eligió esta carretera por presentar tres tramos geométricos claramente diferenciados. El primero de ellos (desde Losa del Obispo hasta el municipio de Chelva) presenta radios relativamente elevados y baja pendiente longitudinal. El segundo tramo (entre Chelva y el municipio de Tuéjar, presenta muchas curvas encadenadas de radios bajos y pendientes longitudinales moderadas. El último tramo, finalmente, cuenta con muchas curvas, menos encadenadas pero de radios todavía menores y pendientes longitudinales muy elevadas.

En cuanto a la sección transversal, en el primer tramo cuenta con ancho de carriles de 3,5 m $\mathrm{y}$ arcenes de al menos $0,5 \mathrm{~m}$, mientras que los dos últimos tramos presenta, en general, carriles de $3,25 \mathrm{~m}$ y $0,25 \mathrm{~m}$ de arcenes.

El tráfico existente en todos los tramos es similar, de aproximadamente unos $2012 \mathrm{veh} / \mathrm{día}$, según el promedio de IMD entre los años 2009 y 2013.

\subsection{Toma de datos en campo}

La toma de datos en campo se realizó entre marzo y abril de 2014. En estas pruebas, los 
conductores circulan por el tramo utilizando su propio vehículo. Cada vehículo se equipa con una serie de cámaras apuntando en diferentes direcciones, según la Figura 1.

Las cámaras utilizadas son VIRB Elite, de Garmin. Se trata de cámaras de acción que permiten grabar en HD y registrar la posición y velocidad (van equipadas con un dispositivo GPS). De esta forma, es posible después restituir las trayectorias, velocidades y percepciones de los conductores.

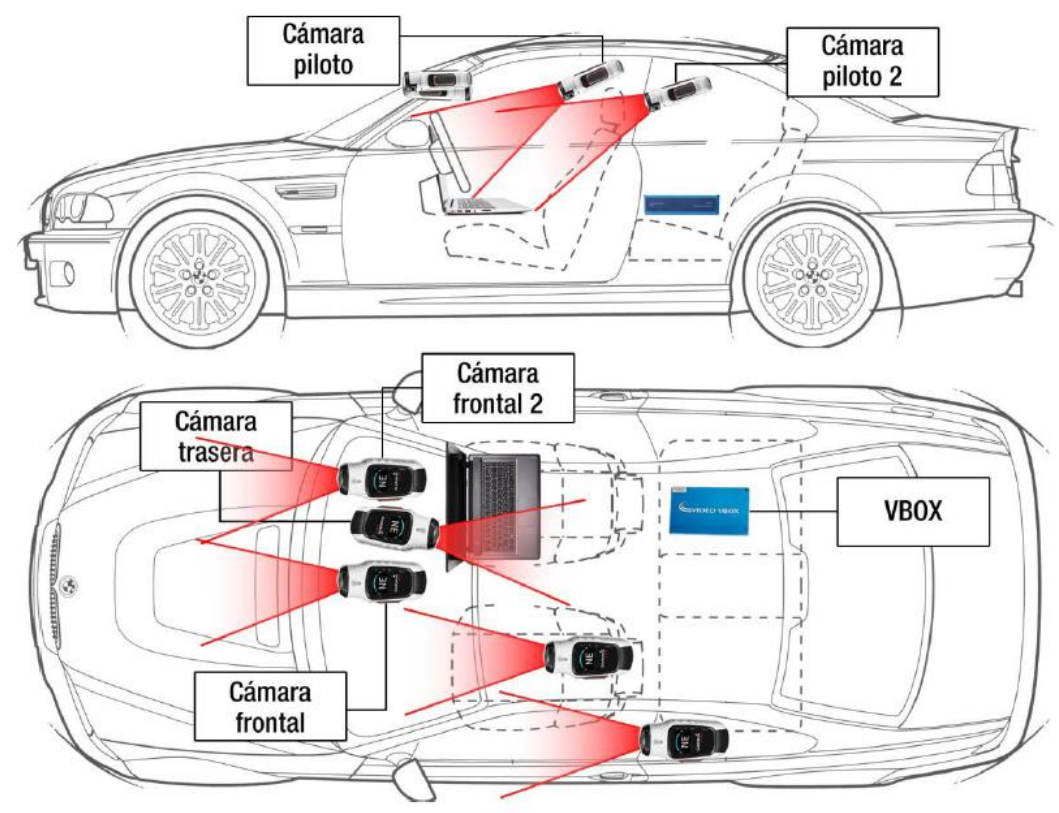

Figura 1. Instrumentación del vehículo para las pruebas.

Igualmente, y con el objetivo de calibrar la fiabilidad de los resultados, algunas pruebas fueron equipadas con un VBOX, GPS de mayor precisión y frecuencia $(10 \mathrm{~Hz})$, que cuenta también con un acelerómetro.

En estas pruebas el conductor iba acompañado de un copiloto y, en ocasiones, de un pasajero adicional. Cada uno de los ocupantes del vehículo debía proporcionar los datos de PSR en cada una de las curvas sin influirse mutuamente, para lo que se llegó a la conclusión de que el mejor método era que el conductor indicase la PSR con los dedos de una mano del volante (sin soltarlo), tras pasar cada una de las curvas (Figura 2).

La escala de PSR va desde 1 (ninguna incomodidad) hasta 5 (gran incomodidad, cercano al deslizamiento del vehículo).

A los conductores no se les informa del objetivo de las pruebas, aunque sí se les describe de forma clara qué deben hacer. Además, se les encomienda a que conduzcan de una forma natural, como habitualmente lo harían. 


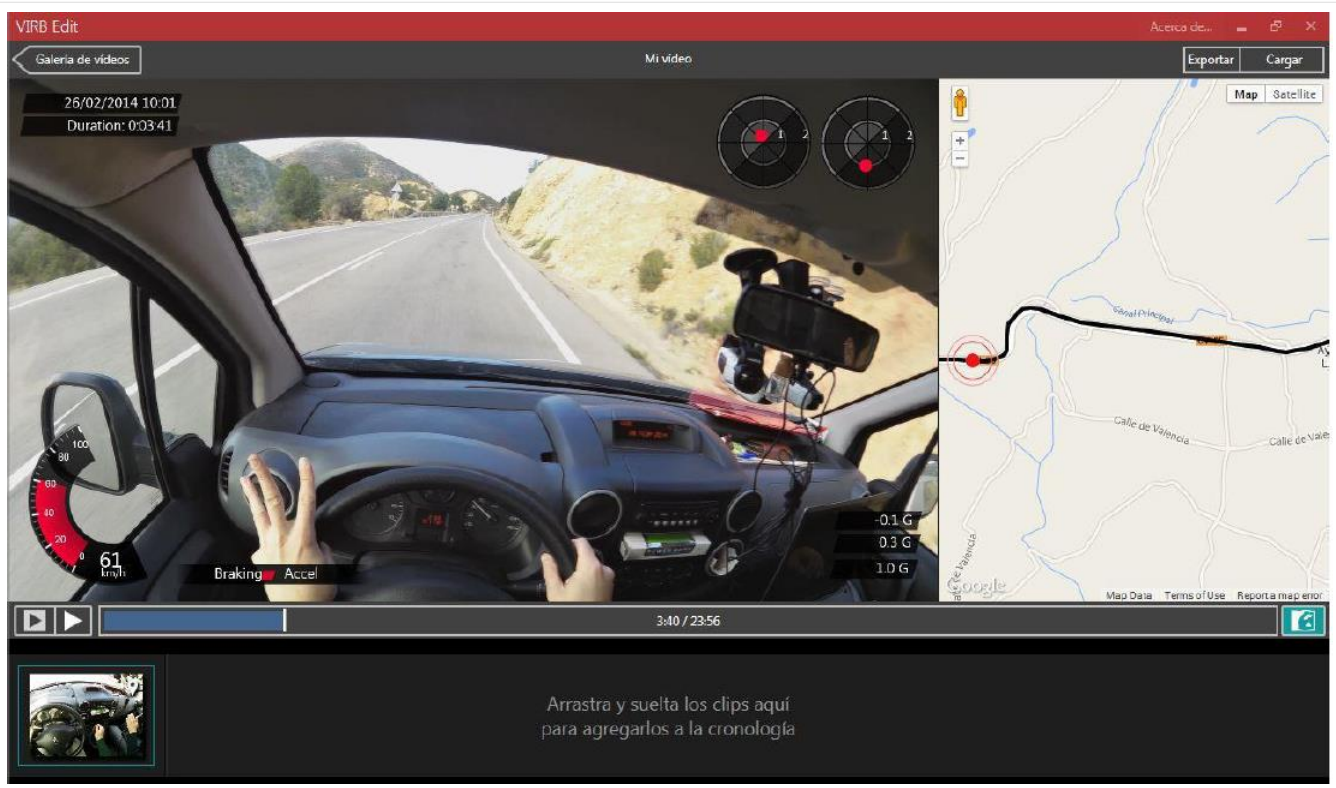

Figura 2. Fotograma en el que se puede observar cómo el conductor indica su PSR tras una curva.

Previamente a cada prueba se realiza una pequeña encuesta en la que se solicitan los datos referentes a:

- Nombre y apellidos

- Edad

- Experiencia al volante (años)

- Kilómetros recorridos en el último año

- Número de veces que ha recorrido el tramo antes

Tras la realización del tramo, nuevamente se les hacen unas preguntas a los conductores:

- ¿Han notado sensación de mareo? Respuesta discreta: nada/bajo/medio/alto.

- ¿Su conducción ha sido natural? Respuesta discreta: nada/poco/medianamente/muy natural.

- ¿Cómo ha sido su carga de trabajo al realizar la prueba? Respuesta discreta: nada/baja/media/alta.

- ¿Le ha influido el tráfico en la elección de la velocidad? Respuesta discreta: nada/poco/medianamente/mucho.

- ¿Ha visualizado el recorrido antes de realizar la prueba? Respuesta discreta: no/poco/medianamente/mucho.

Igualmente, se realiza una fotografía a la ficha técnica del vehículo para disponer de sus características básicas.

28 conductores realizan la prueba de campo. La edad oscila entre 21 y 61 años, con una distribución hombres/mujeres de 80/20. Esta distribución es similar a la existente de conductores de la región, la cual se conocía de un estudio anterior en la zona (Pérez-Zuriaga 
et al., 2010). Estos mismos datos previos fueron utilizados para comparar las velocidades de operación desarrolladas por los sujetos de la prueba con las existentes en la carretera, validando el carácter naturalístico de la prueba (García et al., 2015).

\subsection{Toma de datos en simulador}

La toma de datos en entorno simulado fue realizada en el simulador $\mathrm{SE}^{2} \mathrm{RCO}$, del Instituto de Diseño y Fabricación (IDF) de la Universitat Politècnica de València. Se trata de un simulador compuesto por un ordenador de simulación, recogida de datos en tiempo real, tres pantallas con $120^{\circ}$ de campo de visión, volante, pedales y cambio de marchas de un Citroën Saxo, y asiento ajustable. Este simulador fue validado en cuanto a velocidades de operación, tal y como también se puede ver en Llopis-Castelló et al. (2016).

La carretera y su entorno se simularon empleando software comercial. La restitución geométrica de la misma se hizo con gran fiabilidad gracias a una metodología y software desarrollados por el Grupo de Investigación en Ingeniería de Carreteras (GIIC) de la Universitat Politècnica de València (Camacho-Torregrosa et al., 2015). Tanto el simulador como una captura del entorno real y simulado pueden verse en la Figura 3.
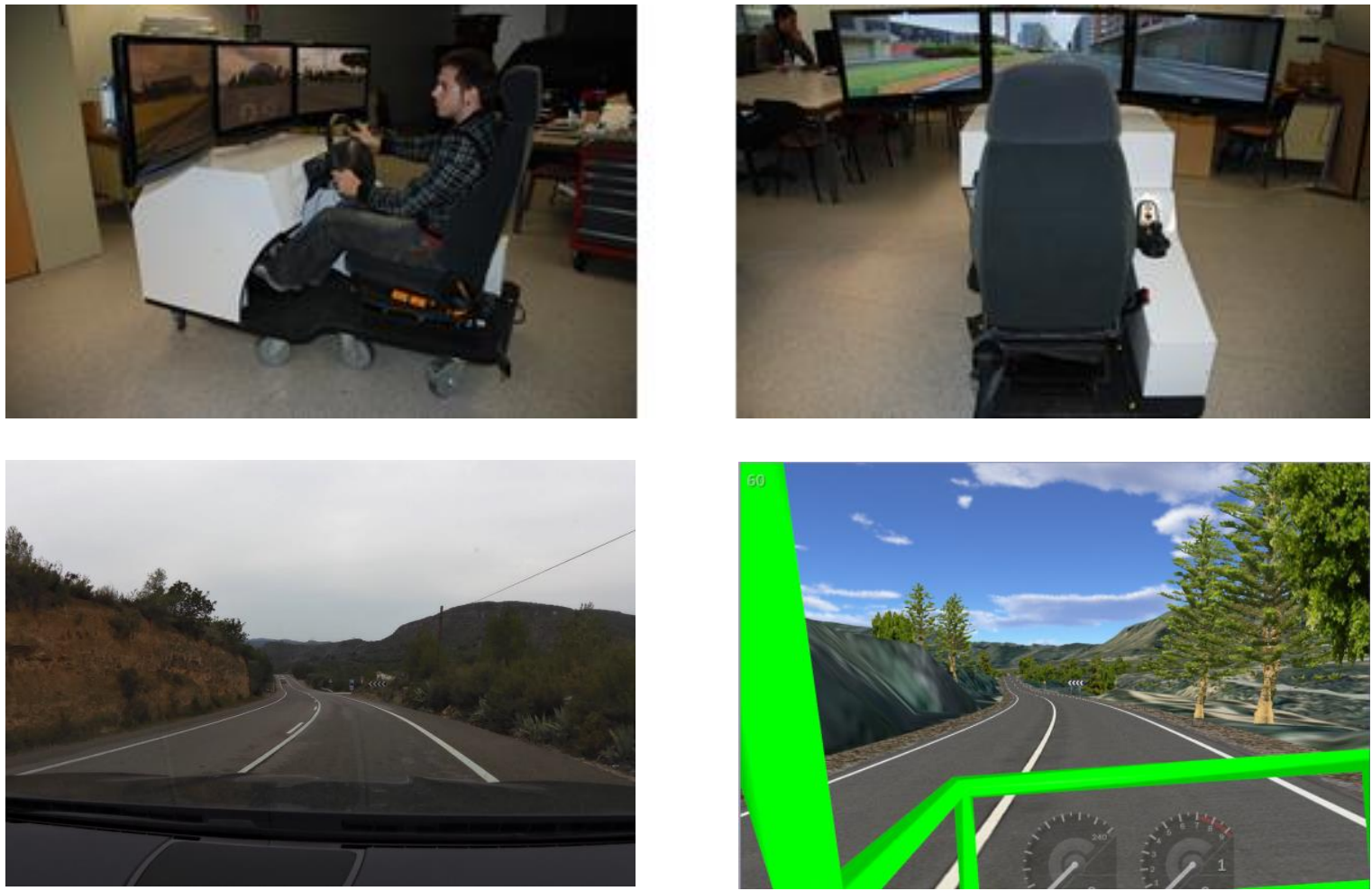

Figura 3. Simulación de conducción en $\mathrm{SE}^{2} \mathrm{RCO}$

Conducir en un entorno simulado puede generar problemas de adaptación, debido a la discordancia entre las percepciones del entorno recibidas por los diferentes sentidos. Además, los sujetos deben realizar un entrenamiento previo para acostumbrarse a la sensibilidad de los mandos del simulador. Así pues, se programaron las pruebas con el 
siguiente esquema:

1. Se les proporciona información a los conductores sobre cómo van a realizar la prueba. Esto incluye la forma de decir los datos. Puesto que ahora únicamente va a ser registrada su percepción y no la de copiloto o pasajero, basta con que la diga en voz alta. Un miembro del equipo de investigación se encargará de registrar dicho valor en el punto exacto.

2. Encuesta previa. Los sujetos responden a las mismas preguntas que en la toma de datos en campo.

3. Entrenamiento. Los sujetos conducen por un tramo similar al de la prueba durante unos 10 minutos.

4. Descanso. El sujeto se levanta, camina y vuelve al simulador únicamente cuando se cree preparado (varios minutos).

5. Conducción en ida. El conductor realiza la conducción en el tramo de ida de la prueba, lo que se estima en unos 30 minutos.

6. Descanso. Similar al anterior.

7. Conducción de vuelta. El conductor realiza el trayecto de vuelta.

8. Encuesta final. En este caso, además de las preguntas de la prueba de campo, también se cuestiona acerca de las sensaciones de mareo, náuseas, cansancio ocular, dolor de cabeza, somnolencia, percepción sobre la realidad del entorno simulado y similitud de la conducción simulada respecto de la real.

Esta prueba la realizaron 24 de los 28 participantes de la primera prueba. Los cuatro restantes no pudieron realizarla debido a motivos de disponibilidad. Asimismo, de los que sí la realizaron, tres presentaron problemas relacionados con mareos y náuseas, por lo que se procesaron únicamente datos de 21 conductores.

\section{PROCESAMIENTO Y ANÁLISIS}

Para cada curva se computa el promedio y la desviación típica de las PSR, tanto en la realidad como en simulación. También se contrasta con sus características geométricas (radio y CCR) y con los percentiles de velocidad de operación.

No se disponen de PSR de todos los conductores en todas las curvas del recorrido debido a:

- Las curvas muy suaves no son percibidas por todos los conductores.

- Situaciones en las que el conductor circula condicionado por tráfico precedente. Puesto que no está desarrollando su velocidad habitual, la percepción de riesgo no se considera al poder estar sesgada.

La Figura 4 muestra el número de conductores que han dado respuesta para cada una de las curvas, mostradas en función de su CCR. Puede observarse cómo, para CCR por encima de $100 \mathrm{gon} / \mathrm{km}$, el número de respuestas se mantiene muy estable, llegando incluso en simulador al máximo de 21 en numerosas ocasiones. Esto no se produce para la toma de datos de campo, donde el condicionamiento de algunas trayectorias reduce la muestra final. 
Para los casos de menos de 100 gon/km, se ve cómo el número de respuestas válidas decrece paulatinamente debido a la menor percepción de las curvas.

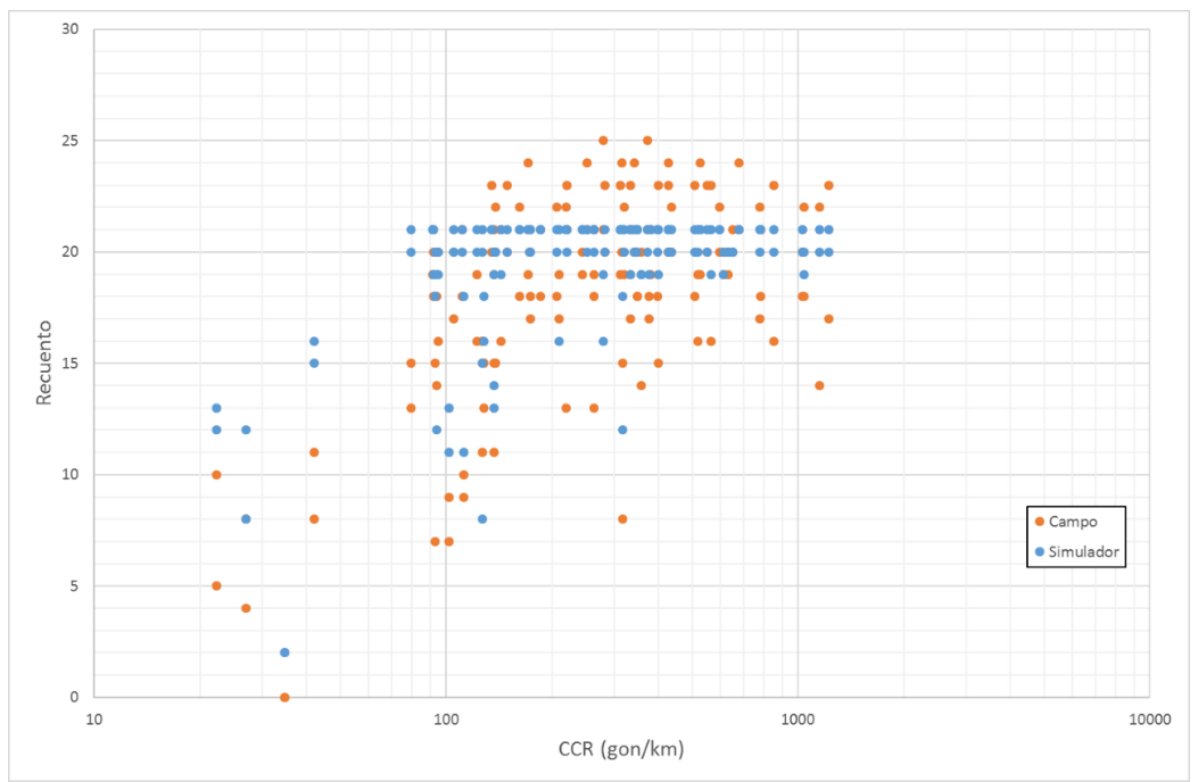

Figura 4. Recuentos en función de CCR.

Si se efectúa una representación gráfica del recuento de respuestas en función del promedio de la PSR observada (Figura 5), puede observarse un comportamiento similar. Curvas con poca percepción de riesgo (promedio inferior a 2) suelen presentar recuentos mucho más dispersos que las curvas con más percepción de riesgo.

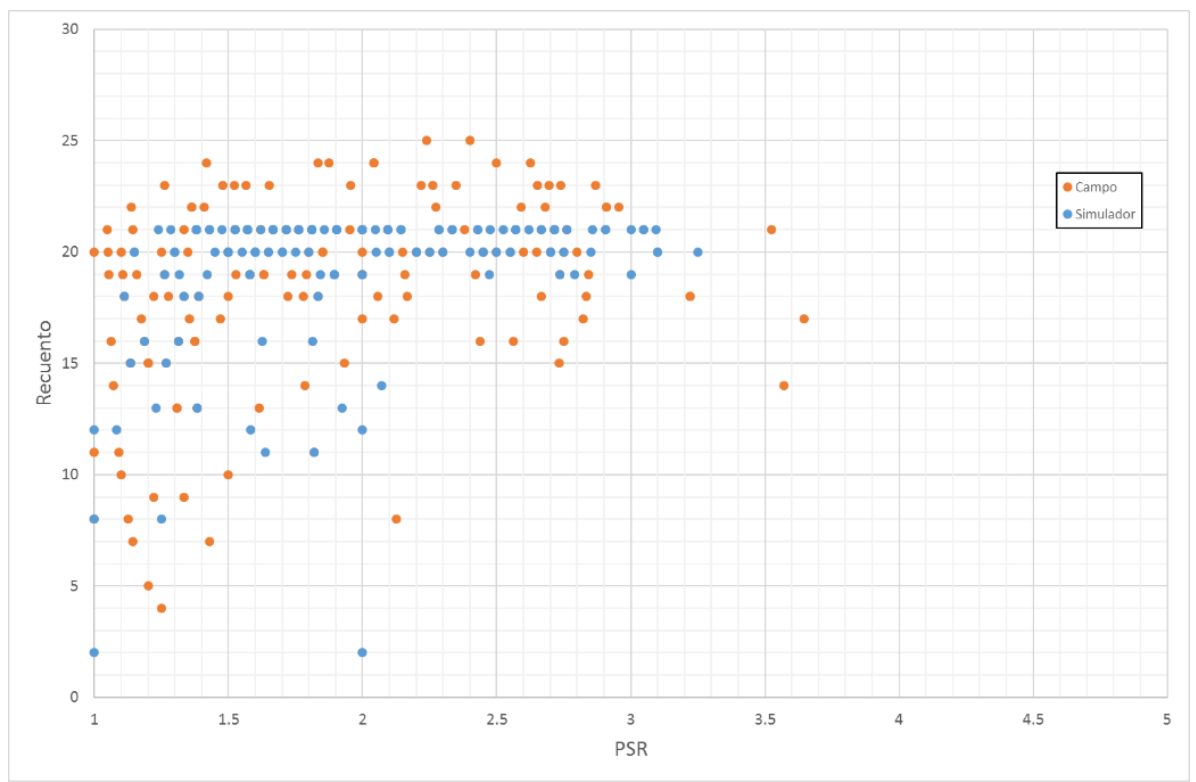

Figura 5. Recuento en función del PSR.

Las curvas con un muy reducido número de respuestas, tanto en la realidad como en simulador, fueron retiradas del análisis. Así, finalmente se cuenta con 146 curvas a analizar contando ida y vuelta. 
Ya con los datos válidos, se ha contrastado la PSR en función del radio y del CCR (Figura 6). Se pueden apreciar los siguientes aspectos:

- La PSR del simulador suele ser algo superior a la recogida en campo. La PSR simulada suele ser menor para curvas cerradas, pero parece ir aumentando su diferencia respecto de la PSR de campo para curvas más abiertas.

- Hay un cambio claro de comportamiento en torno a los 300-400 m de radio. A partir de estos valores, la PSR parece estabilizarse en valores mínimos, esto es, el riesgo percibido ya es independiente de la geometría.

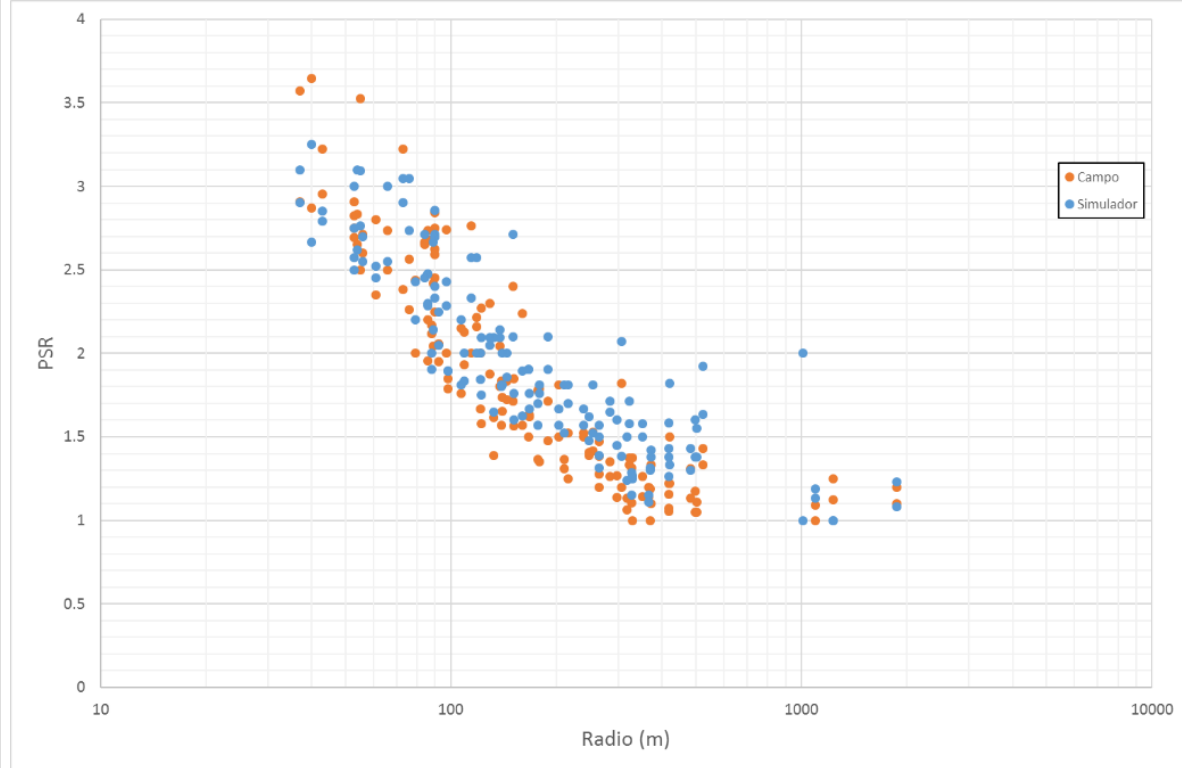

\section{Figura 6. Evolución de la PSR en función del radio.}

La Figura 7 muestra lo mismo, aunque en función de CCR.

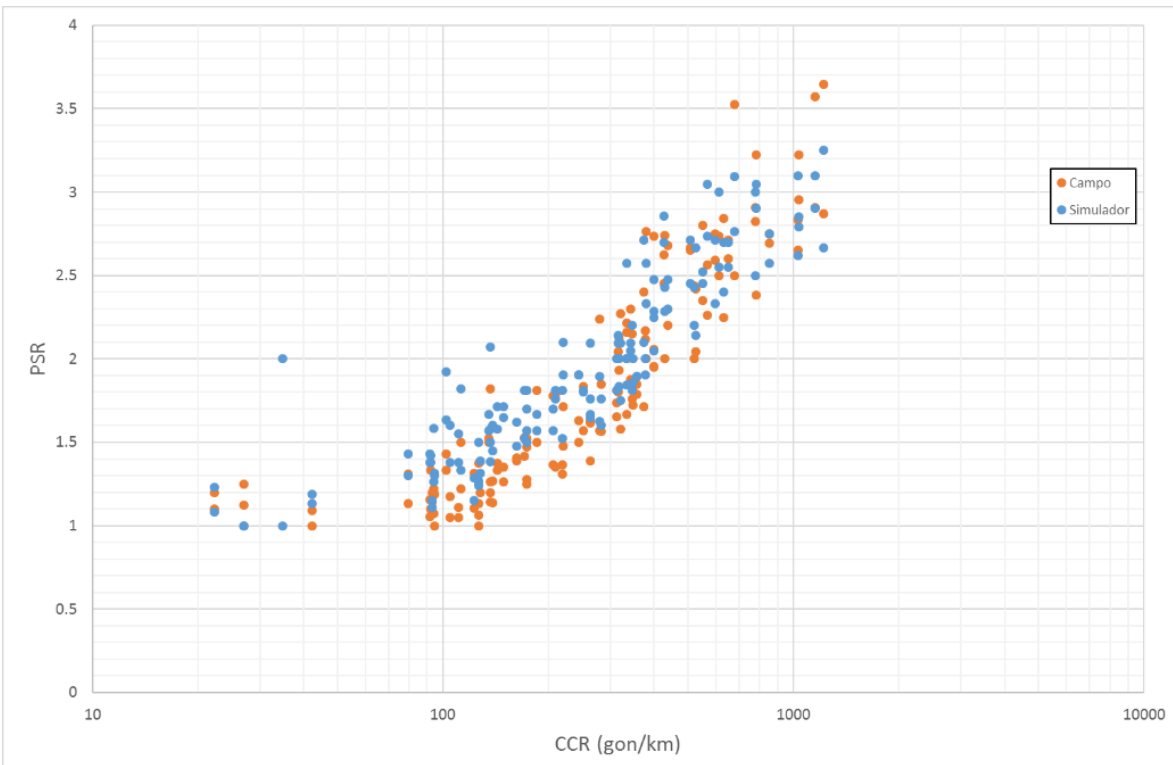

Figura 7. Evolución de la PSR en función del CCR. 
En cuanto a dispersión de las PSR recogidas (expresada como desviación típica entre las repuestas de los conductores), puede verse como es algo superior para los datos del simulador respecto del campo. Esto puede explicarse por la no existencia de aceleraciones transversales (Figura 8).

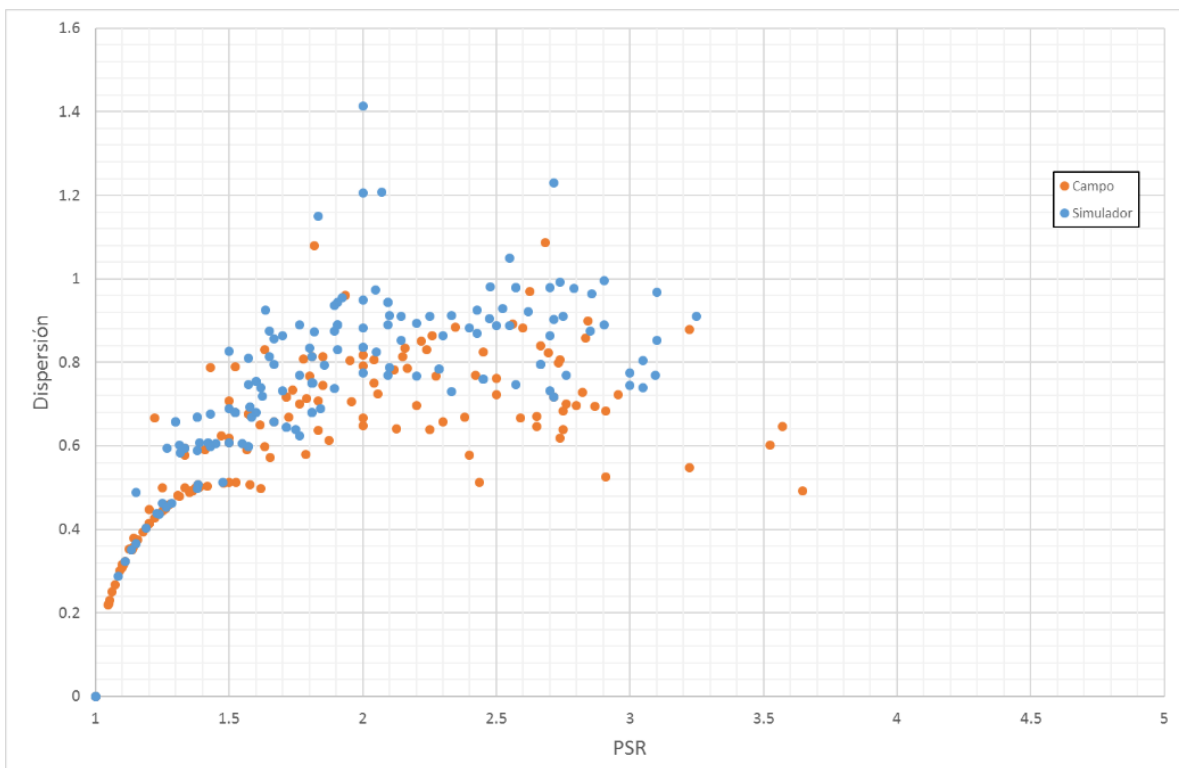

Figura 8. Desviación típica de la PSR recogida en función de dicho valor.

El estudio de la dispersión cobra bastante importancia en este momento, pues se pretende posteriormente calibrar funciones de estimación de la PSR a partir del radio (Figura 9) y del CCR (Figura 10).

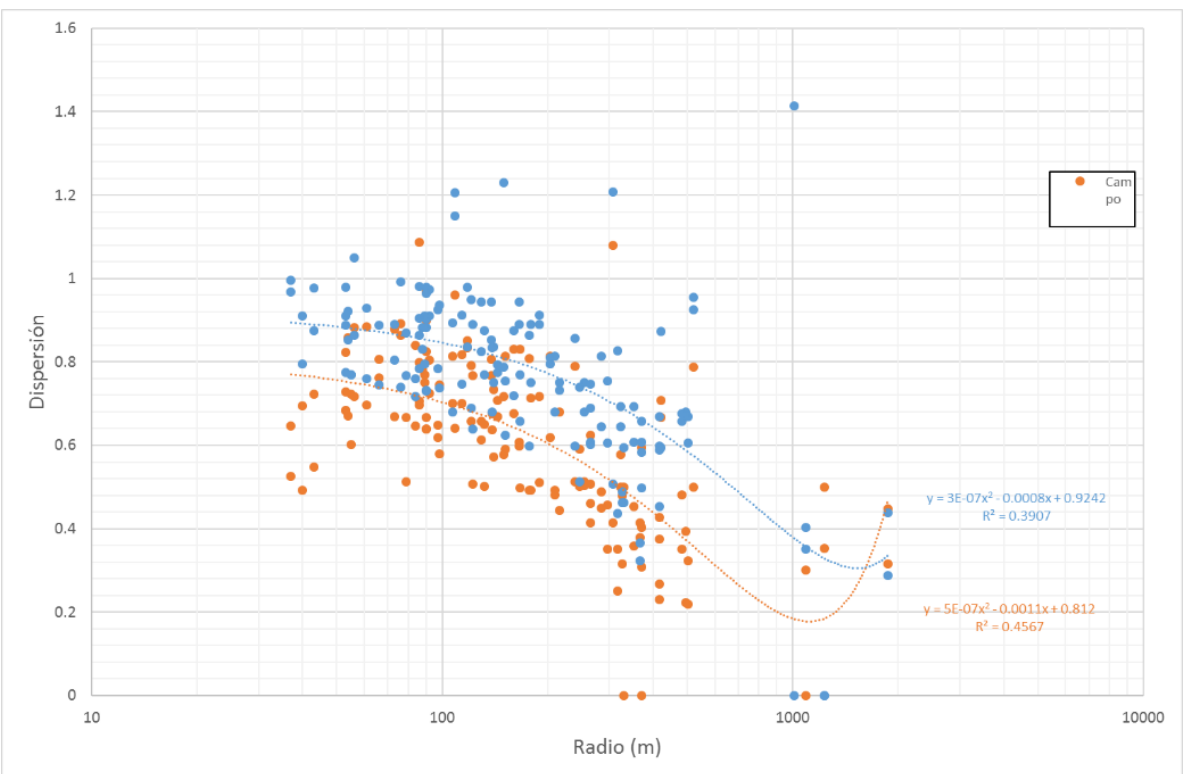

Figura 9. Desviación típica de la PSR en función del radio. 


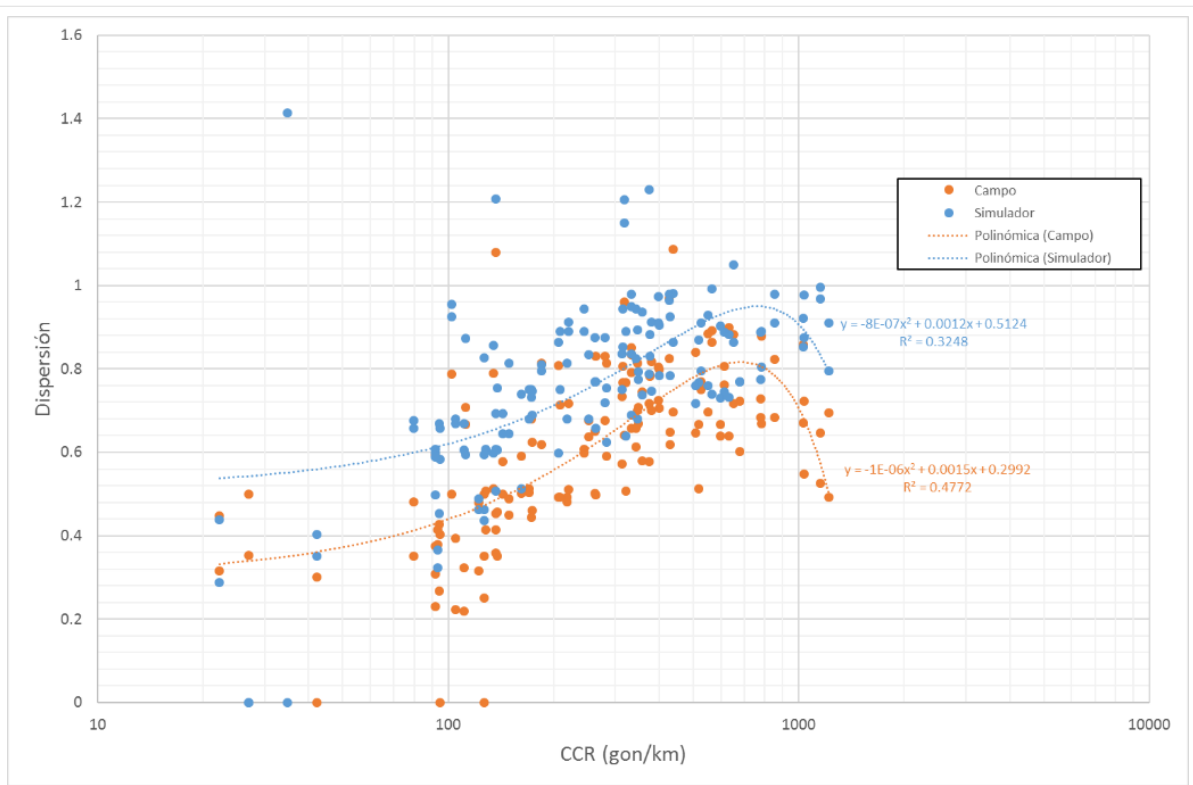

Figura 10. Desviación típica de la PSR en función del CCR.

\section{DISCUSIÓN}

Anteriormente se han presentado los datos obtenidos tras su obtención y un primer filtrado, además de presentar las bases para la estimación de la PSR. En este apartado se va a realizar dicha estimación para condiciones de simulador y campo, así como su comparación.

Se ha realizado un ajuste por máxima verosimilitud del PSR en función de la geometría, tanto para el radio como para el CCR. El ajuste estadístico se ha realizado cogiendo todos los datos válidos a nivel de conductor individual, es decir, sin agrupar por curvas. Como forma funcional, se ha escogido una función logística, pues su comportamiento y recorrido se pueden encajar para la PSR (Ecuación 1).

$$
P S R=\alpha_{0}+\frac{\alpha_{1}}{1+e^{-\beta_{0}-\beta_{1} \cdot X}}
$$

Donde $\alpha_{0}$ es una constante a calibrar que determina el límite inferior de la función, $\alpha_{1}$ es otra constante a calibrar que determina el límite superior de la función, $\beta_{0}$ y $\beta_{1}$ son otras constantes a calibrar, y $X$ es la variable explicativa (radio o CCR según el caso).

La desviación típica en función del radio y CCR se puede obtener a partir de un ajuste (ecuaciones de la Tabla 1), y se introducirá en el ajuste.

\begin{tabular}{|l|c|c|l|}
\hline Radio en campo & $\sigma=5 \cdot 10^{-7} \cdot R^{2}-0,0011 \cdot R+0,812$ & $45,67 \%$ & $(2)$ \\
\hline Radio en simulador & $\sigma=3 \cdot 10^{-7} \cdot R^{2}-0,0008 \cdot R+0,9242$ & $39,07 \%$ & $(3)$ \\
\hline CCR en campo & $\sigma=-10^{-7} \cdot C C R^{2}+0,0015 \cdot C C R+0,2992$ & $47,72 \%$ & $(4)$ \\
\hline CCR en simulador & $\sigma=-8 \cdot 10^{-7} \cdot C C R^{2}+0,0012 \cdot C C R+0,5124$ & $32,48 \%$ & $(5)$ \\
\hline
\end{tabular}

Tabla 1. Expresiones de la desviación típica en función del radio y del CCR. 
Se calibraron funciones para estimar la PSR en función del radio y el CCR, tanto para el entorno real como para el simulado. Los parámetros $\alpha_{0}$ y $\alpha_{1}$ se pudieron obtener del ajuste, pero se prefirió forzarlos a 1 y 4 para no sobreajustar las funciones. De este modo, el resultado de las funciones calibradas está siempre entre 1 y 5 , es decir, en su rango real.

Las funciones ajustadas obtenidas fueron las mostradas en la Tabla 2.

\begin{tabular}{|l|l|l|l|}
\hline Variable explicativa y ámbito & Expresión & Log-lik. & Eq. \\
\hline Radio en campo & $P S R=1+\frac{4}{1+e^{-0,3422+0,011 \cdot R}}$ & $-2598,4$ & $(6)$ \\
\hline Radio en simulador & $P S R=1+\frac{4}{1+e^{0,0606+0,00642 \cdot R}}$ & $-3405,1$ & $(7)$ \\
\hline CCR en campo & $P S R=1+\frac{4}{1+e^{2,4343-0,00276 \cdot C C R}}$ & $-2790,6$ & $(8)$ \\
\hline CCR en simulador & $P S R=1+\frac{4}{1+e^{1,9717-0,002143 \cdot C C R}}$ & $-3457,4$ & $(9)$ \\
\hline
\end{tabular}

Tabla 2. Expresiones de estimación calibradas para el PSR en función del radio y del CCR, para el entorno real y el simulado.

Estas expresiones se muestran en la Figura 11 (en función del radio) y en la Figura 12 (en función del CCR). Se muestran también los intervalos de confianza para el 66\%. No se muestran para un intervalo mayor porque la variabilidad de los datos es tal que llevaría los umbrales hasta casi la totalidad del recorrido de la función.

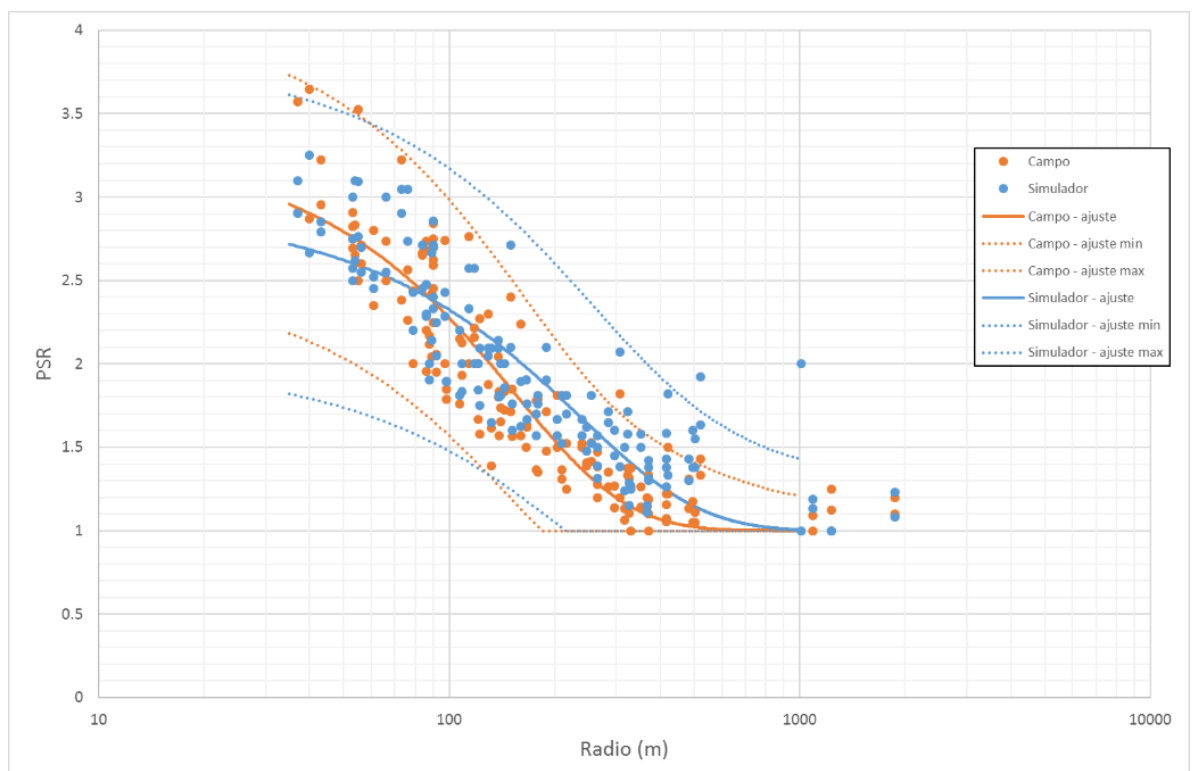

Figura 11. Evolución de la PSR en función del radio. 


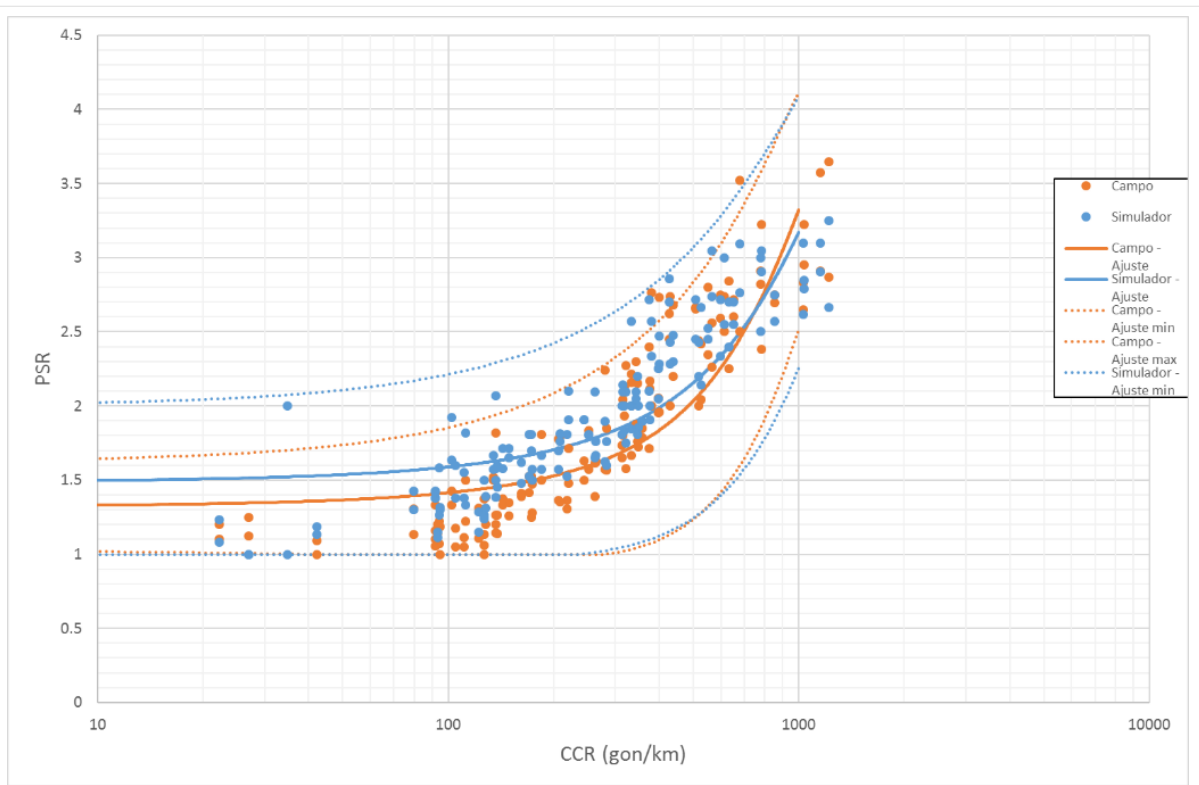

\section{Figura 12. Evolución de la PSR en función del CCR.}

Se puede observar un cambio de comportamiento de la PSR en torno a los $400 \mathrm{~m}$. Este umbral es coincidente con el encontrado por otras investigaciones para el cambio en el comportamiento de la velocidad de operación en curvas (Pérez et al., 2010). La explicación, en ambos casos, parece deberse a que curvas más suaves no ejercen un control geométrico sobre la operación de los conductores, y por lo tanto la velocidad desarrollada por estos pasa a depender de factores subjetivos. Dentro de ellos está la percepción de riesgo, que es por lo general reducida.

Como se ha visto, el ajuste de la PSR en función del radio es un poco más preciso que en función del CCR. Se puede observar, acorde con las primeras observaciones, que la PSR en simulador es ligeramente más tendida que en campo. También los intervalos de confianza son más abiertos en simulador, lo que produce una mayor incertidumbre sobre los resultados.

Finalmente se ha abordado la comparación directa de las PSR obtenidas para campo y para simulador (Figura 13). La relación es bastante similar y próxima a la línea de equivalencia, si bien es ligeramente decreciente. Ello implica que los valores obtenidos en simulación son algo menos sensibles que los de campo: tienen un menor recorrido. 


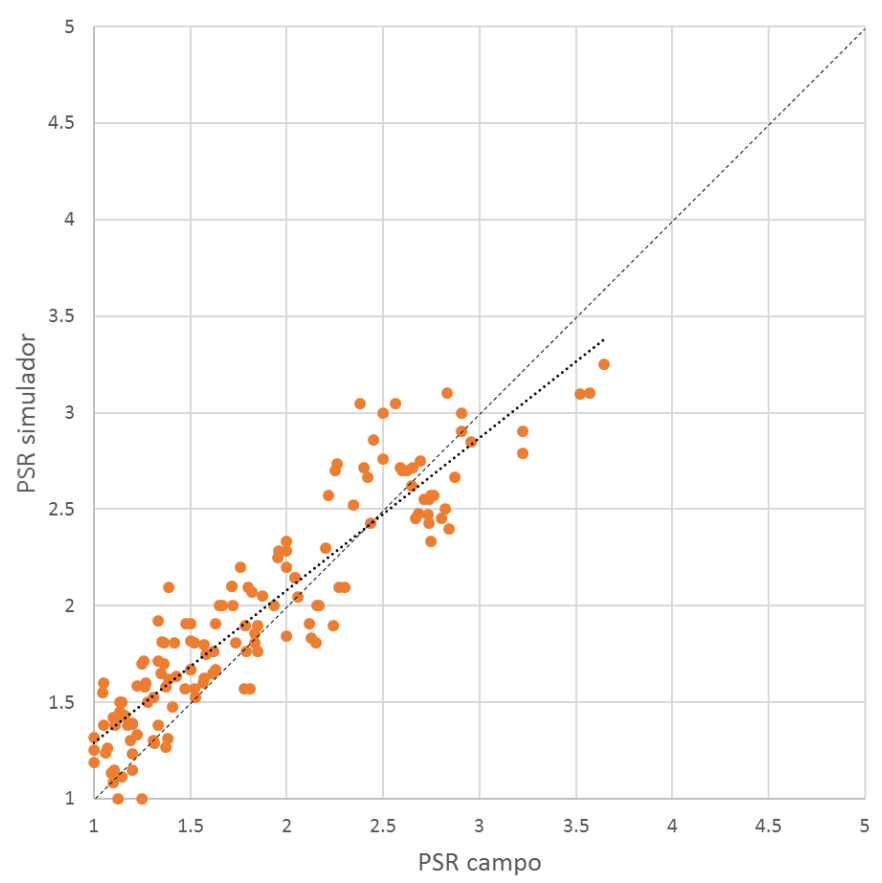

Figura 13. Comparación entre PSR de campo y de simulación.

\section{CONCLUSIONES}

Se ha presentado una investigación que estima la Percepción Subjetiva de Riesgo para curvas en planta (PSR) en función de las características geométricas de las mismas. Las características geométricas son el radio y el CCR, mostrando un ligero mejor ajuste para el primero de ellos.

El conocer estas relaciones permite poder conocer de antemano en un diseño de carreteras qué percepción de riesgo tendrán los conductores, lo cual está relacionado con su consistencia del diseño geométrico y, al fin y al cabo, con su seguridad.

Se ha podido ver cómo tanto en la realidad como en un entorno simulado los conductores son capaces de percibir el riesgo, y que estas percepciones son ligeramente menos precisas en simulación. Sin embargo, la proximidad entre ambas formas de medirla permite utilizar simulación en fase de diseño, arrojando valores de PSR que se corresponderán bastante con los reales.

Como futura investigación, pueden agregarse otras variables operacionales o geométricas a la estimación del PSR, como la velocidad inercial o el trazado en alzado.

\section{AGRADECIMIENTOS}

Los autores quisieran agradecer a la Universitat Politècnica de València (UPV), que financió el proyecto de investigación "CONSIM - Desarrollo de un Modelo para la Evaluación de la Consistencia del Diseño Geométrico de Carreteras Convencionales mediante Simuladores de Conducción" (PAID 05-2012). Asimismo, agradecer también al Ministerio de Economía 
y Competitividad y al Fondo Social Europeo, que financiaron el proyecto de investigación "CASEFU - Estudio experimental de la funcionalidad y seguridad de las carreteras convencionales" (TRA2013-42578-P), del cual forma parte este estudio.

\section{REFERENCIAS}

ARMSBY, P.; BOYLE, A.J. y WRIGHT, C.C. (1989). Methods for Assessing Drivers' Perception of Specific Hazards on the Road. Accident Analysis and Prevention, pp. 45-60. BELLA, F. (2009). Can Driving Simulators Contribute to Solving Critical Issues in Geometric Design? Transportation Research Record: Journal of the Transportation Research Board 2138, pp. 120-126.

CAMACHO-TORREGROSA, F. J., PÉREZ-ZURIAGA, A. M., CAMPOY-UNGRÍA, J. M., GARCÍA, A., y TARKO, A. P. (2015). Use of Heading Direction for Recreating the Horizontal Alignment of an Existing Road. Computer-Aided Civil and Infrastructure Engineering 30(4), pp. 282-299.

CHARLTON, S.G.; STARKEY, N.; PERRONE, J. e ISLER, R. (2014). What's the Risk? A Comparison of Actual and Perceived Driving Risk. Transportation Research Part F: Traffic Psychology and Behavior, 25. Pp. 50-64.

FULLER, R. (2005). Towards a General Theory of Driver Behavior. Accident Analysis and Prevention, 37(3), pp. 461-472.

GARCÍA, A., CAMACHO-TORREGROSA, F. J., y MARÍN-MORALES, J. (2015). Experimental determination of the inertial operating speed for consistency and segmentation analysis. Transportation Research Board 94th Annual Meeting, 15-4079.

KANELLAIDIS, G. y DIMITROPOULOS, I. (1994). Subjective and Objective Evaluation of Risk on Roadway Curves. Traffic Engineering and Control 55:451-454.

KANELLAIDIS, G., ZERVAS, A. y KARAGIOULES, V. (2000). Drivers' Risk Perception of Road Design Elements. Transportation Human Factors 2, pp. 39-48.

KAPTEIN, N., THEEUWES, J. y VAN DER HORST, R. (1996). Driving Simulator Validity: Some Considerations. Transportation Research Record: Journal of the Transportation Research Board 1550, pp. 30-36.

LLOPIS-CASTELLÓ, D. et al. (2016). Validation of a Low-Cost Driving Simulator Based on Continuous Speed Profiles. Transportation Research Record 1441 (in press)

NÄÄTÄNEN, R. y SUMMALA, H. (1976). Road User Behavior and Traffic Accidents. Amsterdam: North Holland

PÉREZ-ZURIAGA, A., GARCÍA, A., CAMACHO-TORREGROSA, F. J., y D’ATTOMA, P. (2010). Modeling Operating Speed and Deceleration on Two-Lane Rural Roads with Global Positioning System Data. Transportation Research Record: Journal of the Transportation Research Board 2171, pp. 11-20. 